\title{
Features of Cutaneous Malignant Melanoma Metastatic to the Retina and Vitreous
}

\author{
Mark P. Breazzano ${ }^{a}$ Ann E. Barker-Griffith ${ }^{b}$ \\ ${ }^{a}$ Department of Ophthalmology, Vanderbilt University, Nashville, Tenn., and ${ }^{b}$ Departments of Ophthalmology and \\ Pathology, State University of New York, Upstate Medical University, Syracuse, N.Y., USA
}

\section{Key Words}

Cutaneous malignant melanoma - Vitreoretinal metastasis . Neovascular glaucoma $\cdot$ Ocular oncology $\cdot$ Histopathology

\begin{abstract}
Background/Aims: To report a case of cutaneous malignant melanoma with cerebral metastasis found to have vitreoretinal metastasis upon referral for neovascular glaucoma. Methods: The clinical history and ocular examination findings, including histologic, cytologic, genetic, and immunohistochemical features of the vitreoretinal metastatic tumor, were reviewed. Additionally, the histologic and immunohistochemical features of the primary skin tumor and brain metastasis were also assessed. Results: A 62-year-old woman with cutaneous malignant melanoma metastatic to the right frontal lobe (BRAF V600E negative) was evaluated for blurred vision in the right eye. Neovascular glaucoma, iritis, and posterior synechiae with no view of the retina or vitreous were evident on examination. Vitreoretinal biopsy and enucleation specimen both showed widespread neoplastic involvement of the retina and residual vitreous strands after vitrectomy. Choroid, trabeculum, and other intraocular structures were devoid of tumor burden. Diagnosis of cuta-
\end{abstract}

neous malignant melanoma metastatic to the retina and vitreous was confirmed, and the patient expired shortly thereafter. Conclusion: Cutaneous malignant melanoma metastatic to the eye has a relatively greater preference for the retina and frequently presents with uveitis and glaucoma. Neovascular glaucoma in these cases may likely be attributable to unusually increased vascular endothelial growth factor production by the intraocular melanoma tumor cells.

(c) 2015 S. Karger AG, Basel

\section{Introduction}

Cancer metastasizing to the retina is exceedingly rare, yet cutaneous malignant melanoma has a slightly higher propensity to do so than virtually all other neoplasms [1]. Once metastatic inside the eye, cutaneous malignant melanoma often presents with neovascular glaucoma, without a comprehensive explanation to date [2]. Herein, we explore the features of a rare case of cutaneous malignant melanoma with intraocular metastases, exclusively in the retina and vitreous, and hypothesize the stimulus for iris angiogenesis in order to further characterize the pathogenesis of this malignancy.

\section{KARGER}

E-Mail karger@karger.com

www.karger.com/oop
C 2015 S. Karger AG, Base

2296-4681/15/0022-0080\$39.50/0
Ann E. Barker-Griffith, MD, FRCSC

Departments of Ophthalmology and Pathology

State University of New York, Upstate Medical University

766 Irving Avenue, Syracuse, NY 13210 (USA)

E-Mail barkerga@upstate.edu 

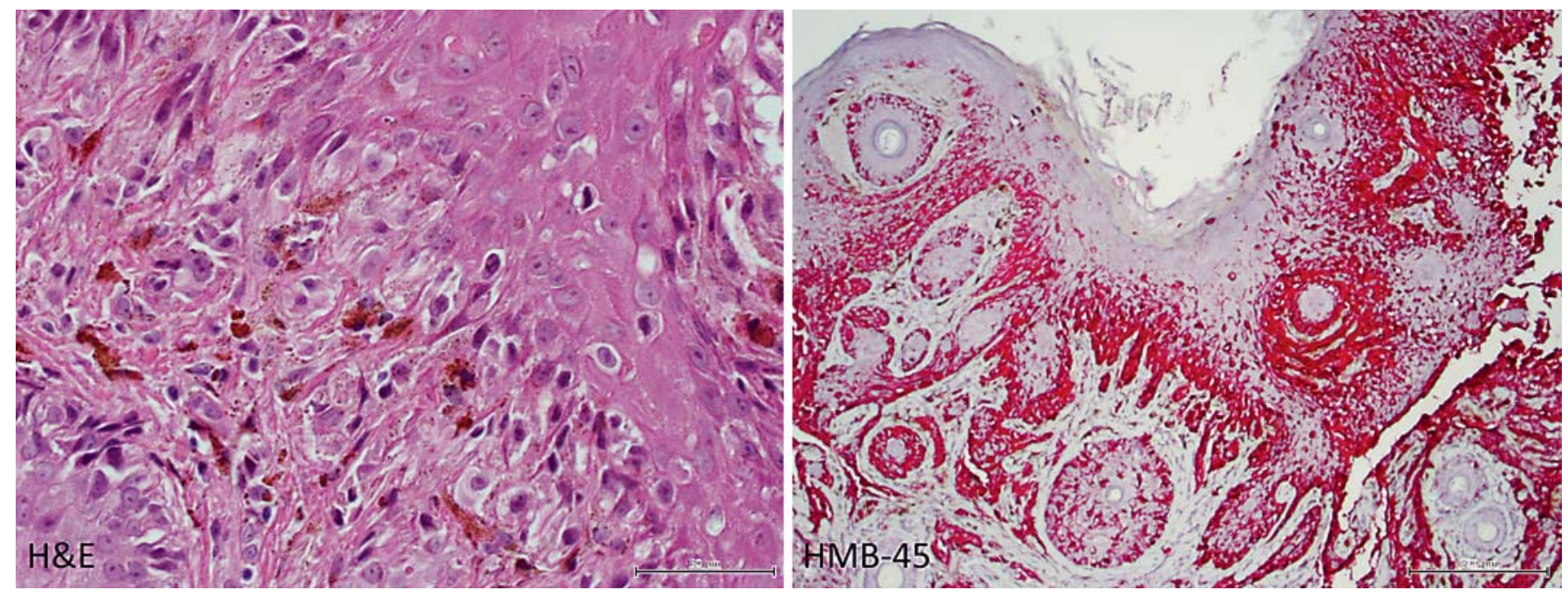

Fig. 1. Histopathology of excised primary skin lesion from the mid-lateral nose. Left: pigmented melanoma cells invade the dermis with epithelial upward migration $(\times 400, \mathrm{HE})$. Right: malignant cells in the epithelium and dermis are positive using a red-chromogen melanocytic immunostain $(\times 100$, HMB-45).
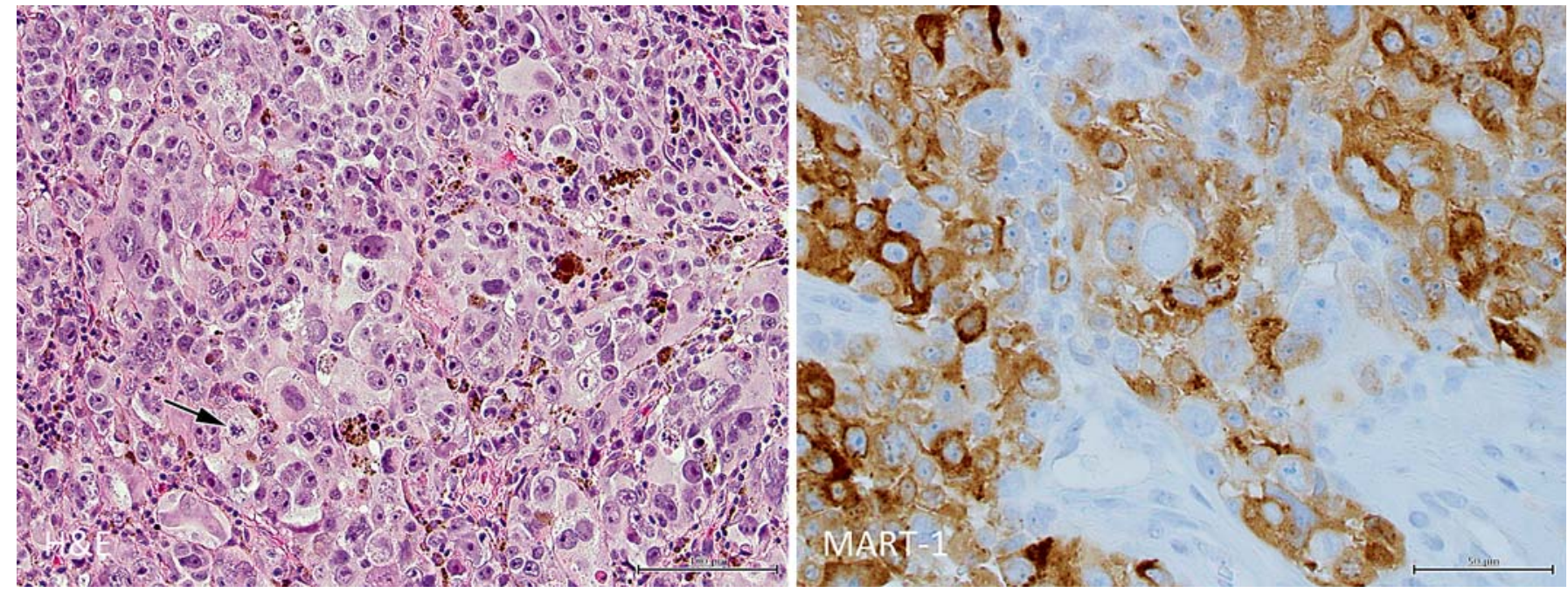

Fig. 2. Histopathology of brain from right frontal lobe neoplasm consistent with metastatic cutaneous malignant melanoma. Left: malignant melanoma cells of varying size with enlarged nuclei and nucleoli show sporadic pigment granules and occasional mitoses (arrow; $\times 200$, HE). Right: malignant cells react to melanocyte marker $(\times 400$, MART -1$)$.

\section{Case Report}

A 62-year-old woman presented to an ophthalmologist with floaters, light flashes, photophobia, and decreased vision in the right eye in May 2014. On examination of the right eye, vision was hand motions, pressure was elevated, and there was protein flare upon slit-lamp biomicroscopy and florid iris neovascularization. There were also posterior synechiae and angle closure with neovascularization. The left eye was normal. There was no improvement with topical brimonidine/timolol and difluprednate as well as oral acetazolamide medications. When the patient's condition did not improve, she was referred to a retina specialist in Syracuse, N.Y. for evaluation of vitritis and neovascular angle-closure glaucoma. The patient's history included excision of a pigmented lesion from the skin of the mid-lateral nose 3 years previously. Histopathologic examination of the excised lesion confirmed cutaneous malignant melanoma (fig. 1). There was compound melanocytic proliferation of irregularly distributed single and nested atypical melanocytes with upward migration. Additionally, there was evidence of invasion of the reticular dermis (Clark's level IV) to a 


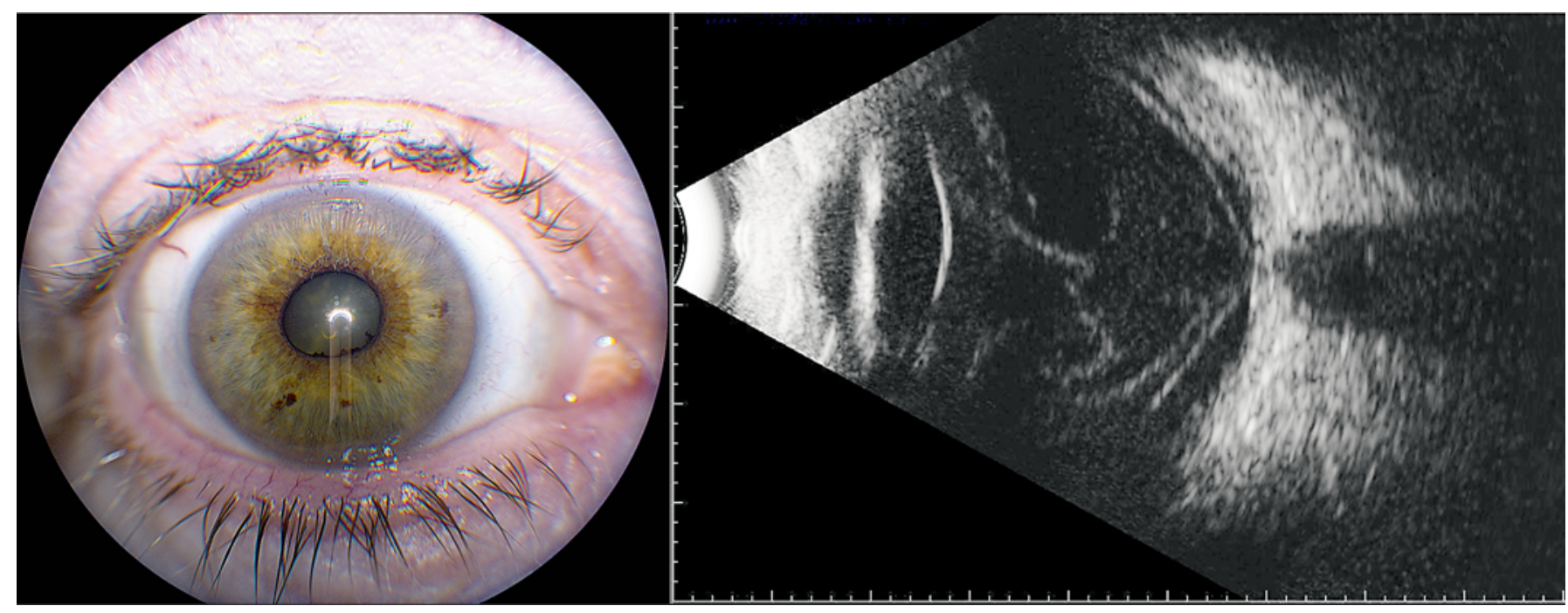

Fig. 3. Clinical imaging of the affected eye. Left: photograph demonstrates posterior synechiae inferonasally. Right: ultrasound shows vitreous opacities and membranes, but without mass lesion or retinal detachment.
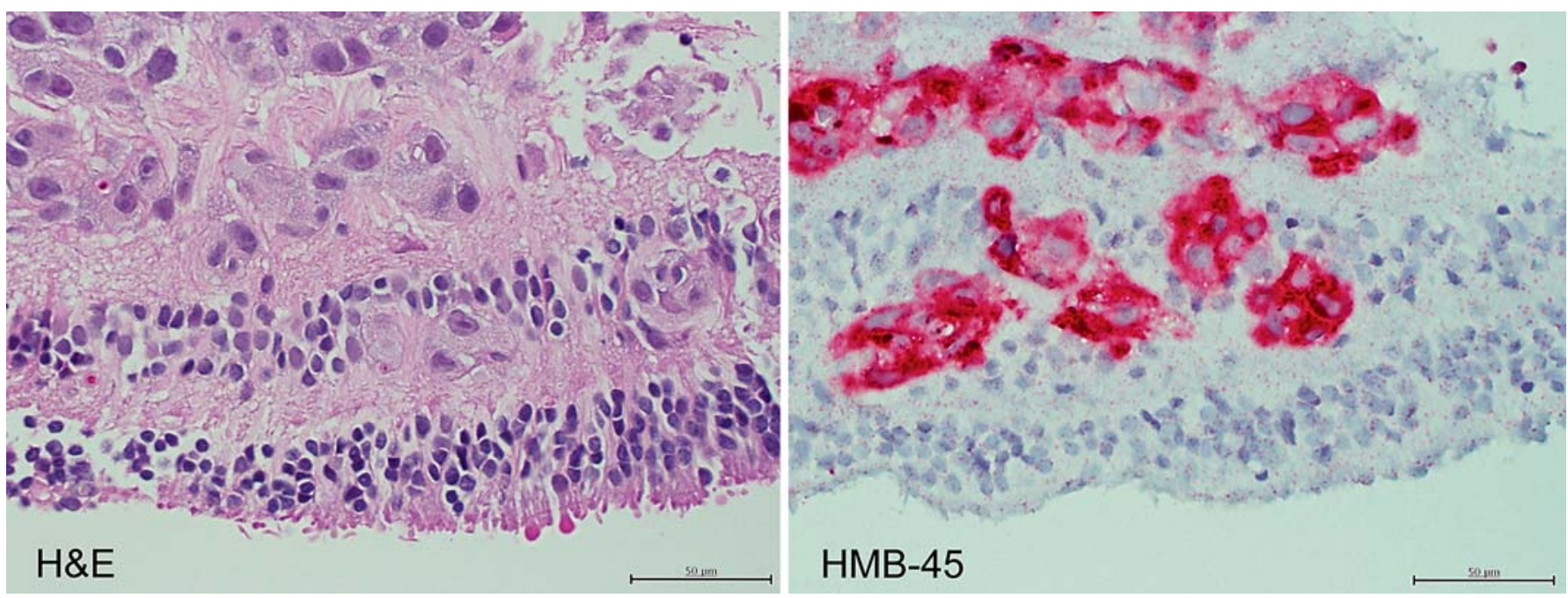

Fig. 4. Histopathology of retinal biopsy from the affected eye. Left: amelanotic, dis-cohesive malignant cells with enlarged nuclei are scattered throughout the retina, most prominently in the inner layers $(\times 400, \mathrm{HE})$. Right: malignant cells are replacing the inner retina $(\times 400$, HMB- 45$)$.

Breslow's depth of $1.25 \mathrm{~mm}$, and all margins were positive. There were less than 1 mitosis per $\mathrm{mm}^{2}$ and no ulceration. Metastatic disease was detected 2 years after the skin biopsy because of seizures. Right frontal lobe tumor excision showed malignant cells positive for MART-1 and S-100 and negative for pancytokeratin (fig. 2). The brain tissue was sent for BRAF mutation assay, which was negative for the BRAF V600E mutation.

On ocular examination in June 2014, visual acuity of the right eye remained hand motions. There was no afferent pupillary defect. The pressure was elevated. The anterior segment showed keratitic precipitates and rubeosis of the iris. There was no view of the vitreous or retina, and the B-scan showed vitreous opacities but no retinal detachment and no mass lesion (fig. 3 ). She was treated with steroids and 2 bevacizumab injections. Improvement did not occur, requiring a vitrectomy for cytopathology and a retinal biopsy (fig. 4). The vitreous cytopathology showed large, pleomorphic, single malignant cells and a few loosely cohesive seeds of these cells with enlarged nuclei, macronucleoli, and a moderate amount of cytoplasm. Multinucleated forms, intranuclear vacuoles, and mitotic figures were noted. Immunohistochemical studies showed the neoplastic cells positive for S-100 and HMB-45. The diagnosis was consistent with metastatic melanoma. The retinal biopsy 


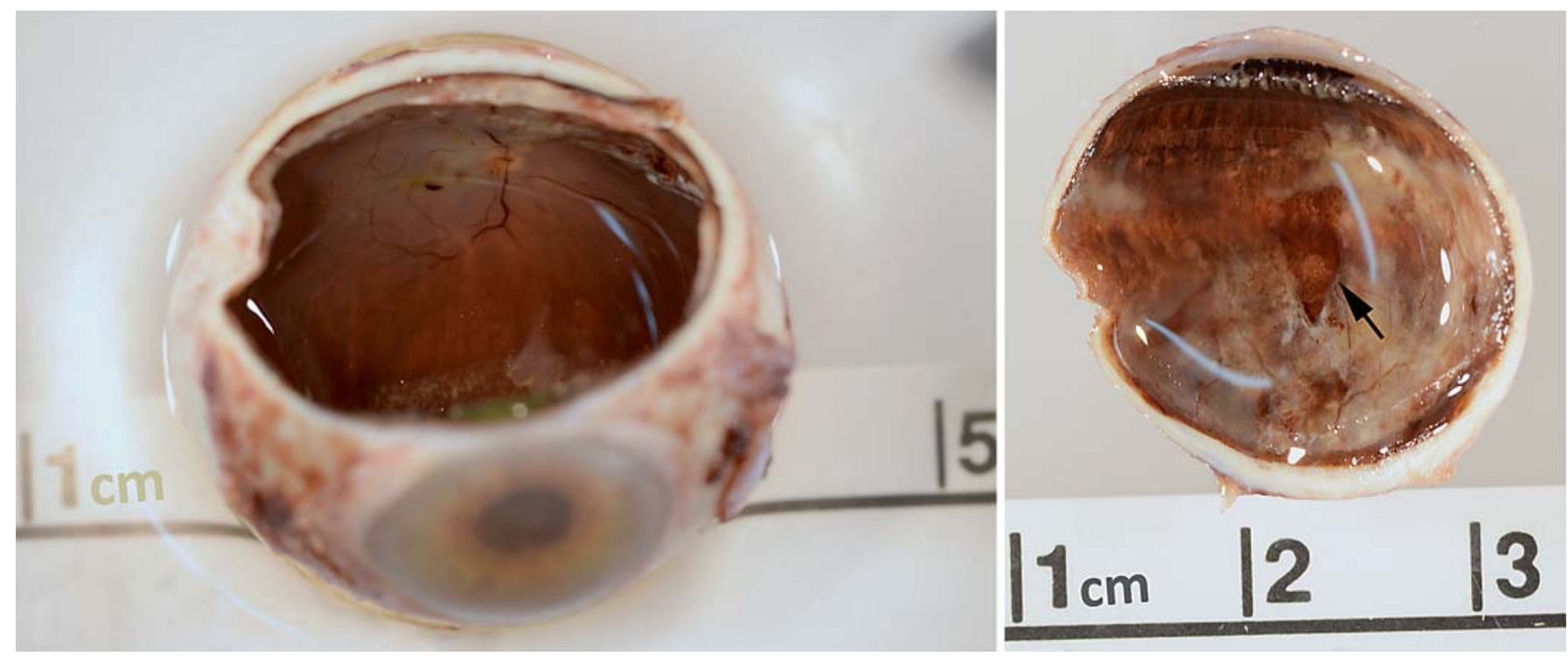

Fig. 5. Gross pathology of the enucleated eye. Left: sectioned globe shows macular hemorrhage and thickened retinal edge. Right: superior calotte shows thickened retina extending to the periphery along with a clear area consistent with the prior vitreoretinal biopsy site (arrow).
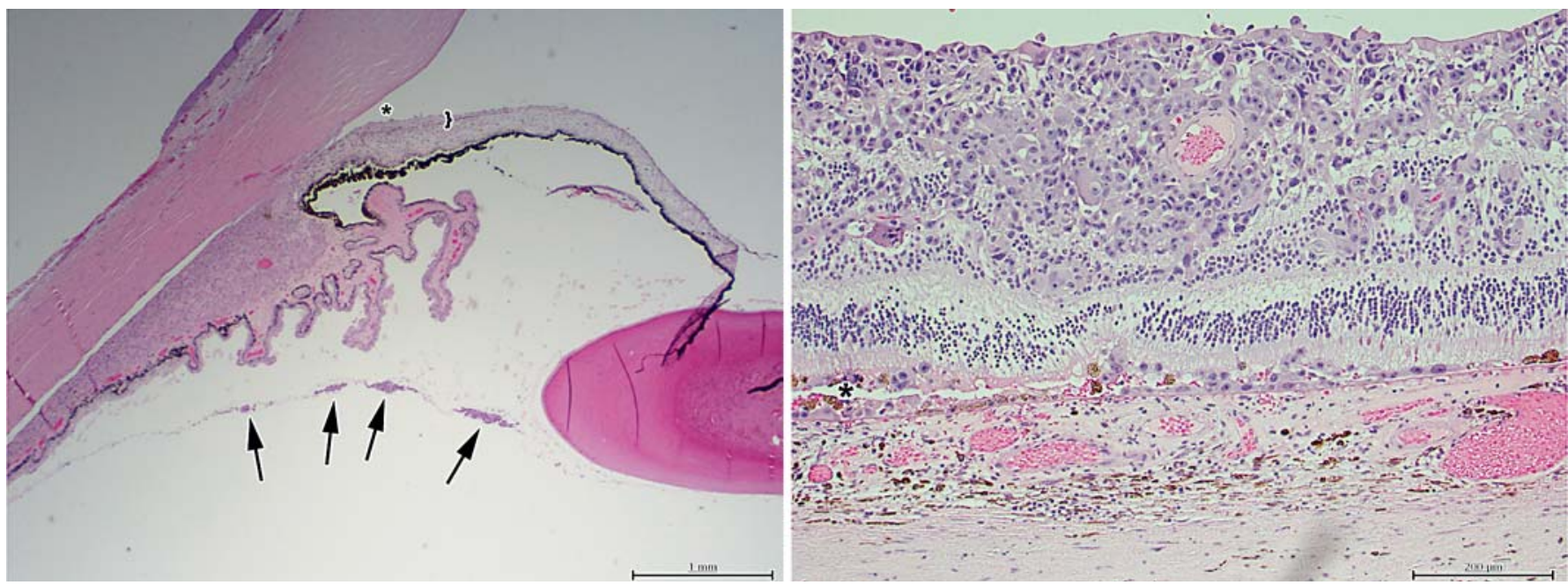

Fig. 6. Histopathology of the enucleated eye (HE). Left: anterior hyaloid vitreous seeds of melanoma cells are shown (four arrows), along with angle closure (asterisk) secondary to iris neovascularization (bracket, $\times 20)$. Right: amelanotic melanoma cells invade the retina and subretinal space (asterisk) while sparing the choroid $(\times 100)$.

showed fragments of outer retina, consisting of photoreceptors, outer plexiform layer, and a portion of inner nuclear layer. The inner retina was replaced by malignant cells. The cells were large and pleomorphic with large nuclei and nucleoli. Abundant cytoplasm did not show pigment. Cells were positive for HMB-45 and S-100 for a diagnosis of metastatic malignant melanoma of the inner retina. Because of the inability to control the pain of neovascular glaucoma, an oncologist recommended enucleation for palliation (fig. 5). The patient subsequently died shortly thereafter.
Histopathology of the eye showed residual clusters of malignant cells in the vitreous and focal invasion of the retina, subretinal space, and on the optic disc (fig. 6). Sections also showed secondary neovascular closed-angle glaucoma without tumor cells in the iris or choroid, cataract, and focal nodular drusen. The malignant cells in the vitreous and retina were similar to those in the retina biopsy showing large, amelanotic, pleomorphic cell clusters with large nucleoli and with numerous mitoses. Immunohistochemical reactivity to HMB-45, MART-1, and S-100 were present. 


\section{Discussion}

Metastases to the eye rarely involve the retina, comprising less than $1 \%$ of cases [1]. For most systemic cancers, the choroid is the most frequent site for intraocular invasion given its prominent vasculature. Cutaneous malignant melanoma spreading to the eye, although still rare, instead presents relatively more frequently with vitreoretinal involvement, with some claiming as high as $49 \%$ of intraocular metastases [2]. It still remains unclear as to the predilection of cutaneous melanoma metastases for retina and vitreous [3]. One suggestion includes trespassing via the optic nerve into the vitreous cavity, consistent with at least one previous report of optic nerve head involvement [4]. Although tumor cells were found on top of the optic disc in our case, this hypothesis may be less likely, given that the tumor cells remained superficial to the nerve, and less suggestive of a thorough, nerve-invading process. Others postulate that the cells are so small, like septic emboli, that they could readily pass into the retinal circulation [5], or that the cells pass directly through the pars plana into the vitreous [2]. Remarkably, the central nervous system is considered the most frequent site of metastasis after other cutaneous/subcutaneous tissues and lymph nodes; nearly half of patients with vitreous metastases have cerebral metastases eventually diagnosed [3]. This pattern is contrary to cutaneous melanoma cases without ocular involvement that more often prefer bone. Interestingly, compared to the rest of the body, primary cutaneous melanomas arising from the head and neck, as in this case, were the least common to metastasize to ocular structures [2]. Future studies may be helpful in elucidating a biological or cellular component involved with the variation in metastatic patterns.

The chief complaint of decreased vision along with floaters in this case are both typical symptoms of cutaneous melanoma metastatic to the vitreous [2]. In about $60 \%$ of cases, 'golden brown spherules' appear in the vitreous early on, before obscuration of the clinician's view; the remaining cases, like this one, have amelanotic cells, resulting in white, nonpigmented clumps [3]. Examination findings of posterior synechiae, secondary iritis, and increased intraocular pressure, which prompted referral to the retina specialist, are also consistent with these previous cases.

Glaucoma has been found in over half of patients with intraocular metastases of cutaneous melanoma [2], much of which can be accounted for by secondary open-angle glaucoma from direct tumor burden and pigmentation. However, between a quarter [2] and two fifths specifically develop neovascular glaucoma [6]. In our case, there was no evidence of melanoma cells or pigment within the angles. Rather, there was increased intraocular pressure attributable to neovascular glaucoma, refractory to antiangiogenic intravitreal therapy. No known cause of increased angiogenesis was present, such as diabetes or a retinal detachment, other than the metastatic melanoma itself, a dilemma raised by previous authors $[5,7]$.

It is our suspicion that the cutaneous malignant melanoma in this case is providing the impetus for neovascular glaucoma through the vascular endothelial growth factor (VEGF) pathway. It has been shown that Breslow's depth of cutaneous malignant melanoma not only correlates with aggressiveness of the tumor, but also with the amount of neoangiogenesis, as quantitatively demonstrated by VEGF expression of the tumor [8]. Our case with a Breslow's depth of $1.25 \mathrm{~mm}$ corresponds to melanoma in the vertical growth phase (greater than $0.76 \mathrm{~mm}$ ) with relatively increased VEGF indices. Recent studies have also shown that the elevated level of VEGF production in malignant melanoma is likely related to production by transformed melanocytes rather than secondary to hypoxia from tumor growth $[8,9]$. Thus, the combination of a relatively deep and aggressive primary skin melanoma, along with the metastasis in the eye, most likely allowed for copious VEGF production and consequential neovascular glaucoma.

Molecular studies have also demonstrated great utility in regards to prognosis with genetic analysis. Patients with a mutation in the BRAF V600E gene have shown a great response to vemurafenib, a B-Raf kinase inhibitor, illustrating one example of recent breakthroughs in diagnosis and treatment [10]. In our case, the BRAF mutation was found negative, and therefore, vemurafenib treatment was deferred. Unfortunately, the prognosis is grave once ocular metastases are found $[1,7]$, with these newest treatments offering only several months of additional life expectancy at best [11].

In summary, cutaneous malignant melanoma metastatic to ocular tissue has relatively greater preference for retina and frequently presents with uveitis and glaucoma. Neovascular glaucoma in these cases may likely be secondary to abnormally increased VEGF production by the metastatic intraocular tumor cells. Future cellular and molecular investigations may help shed further light on these peculiar features of cutaneous malignant melanoma.

\section{Acknowledgements}

Support was funded by unrestricted grants from Research to Prevent Blindness Inc., New York, N.Y., USA and Lions District 20-Y1, Syracuse, N.Y., USA.
Breazzano/Barker-Griffith 


\section{Statement of Ethics}

The State University of New York Upstate Medical University Institutional Review Board did not require review of this study because case reports do not meet the definition of human subject research.

\section{References}

1 Shields CJ, McMahon JF, Atalay HT, Hasanreisoglu M, Shields JA: Retinal metastasis from systemic cancer in 8 cases. JAMA Ophthalmol 2014;132:1303-1308.

2 Rosenberg C, Finger PT: Cutaneous malignant melanoma metastatic to the eye, lids, and orbit. Surv Ophthalmol 2008;53:187-202.

3 Jaissle GB, Szurman P, Rohrbach JM, Gelisken F, Bartz-Schmidt KU: A case of cutaneous melanoma metastatic to the vitreous cavity: possible pathomechanism and review of the literature. Graefes Arch Clin Exp Ophthalmol 2007;245:733-740.

4 Pollock SC, Awh CC, Dutton JJ: Cutaneous melanoma metastatic to the optic disc and vitreous. Arch Ophthalmol 1991;109:13521354.

5 Cole EL, Zakov ZN, Meisler DM: Cutaneous malignant melanoma. Arch Ophthalmol 1986;104:98-101.

\section{Disclosure Statement}

The authors indicate no financial conflict of interest involved in this study.
6 Gupta S, Blair MP, Lin AY, David O, Tessler $\mathrm{HH}$, Goldstein DA: Cutaneous melanoma metastatic to the vitreous cavity. Retin Cases Brief Rep 2011;5:197-200.

7 Gunduz K, Shields JA, Shields CL, Eagle RC Jr: Cutaneous melanoma metastatic to the vitreous cavity. Ophthalmology 1998;105:600605.

8 Rajabi P, Neshat A, Mokhtari M, Rajabi MA, Eftekhari M, Tavakoli P: The role of VEGF in melanoma progression. J Res Med Sci 2012; 17:534-539.

9 Gille J: Antiangiogenic cancer therapies get their act together: current developments and future prospects of growth factor- and growth factor receptor-targeted approaches. Exp Dermatol 2006;15:175-186.
10 Sosman JA, Kim KB, Schuchter L, Gonzalez R, Pavlick AC, Weber JS, McArthur GA, Hutson TE, Moschos SJ, Flaherty KT, Hersey P, Kefford R, Lawrence D, Puzanov I, Lewis KD, Amaravadi RK, Chmielowski B, Lawrence HJ, Shyr Y, Ye F, Li J, Nolop KB, Lee RJ, Joe AK, Ribas A: Survival in BRAF V600-mutant advanced melanoma treated with vemurafenib. N Engl J Med 2012;366:707-714.

11 McArthur GA, Chapman PB, Robert C, Larkin J, Haanen JB, Dummer R, Ribas A, Hogg D, Hamid O, Ascierto PA, Garbe C, Testori A, Maio $\mathrm{M}$, Lorigan $\mathrm{P}$, Lebbe $\mathrm{C}$, Jouary $\mathrm{T}$, Schadendorf D, O’Day SJ, Kirkwood JM, Eggermont AM, Dreno B, Sosman JA, Flaherty KT, Yin M, Caro I, Cheng S, Trunzer K, Hauschild A: Safety and efficacy of vemurafenib in BRAF(V600E) and BRAF(V600K) mutation positive melanoma (BRIM-3): extended follow-up of a phase 3 , randomized, open-label study. Lancet Oncol 2014;15:323-332. 